\title{
NUMERICAL MODELING OF GAS TURBINE COOLED BLADES
}

\author{
A.M. Pashayev, D.D. Askerov, R.A. Sadiqov, A.S. Samedov
}

Azerbaijan National Academy of Aviation. AZ-1045,Bina, 25 th $\mathrm{km}$, Baku, Azerbaijan. E-mail: sadixov@mail.ru Received 1412 2004, accepted 29082005

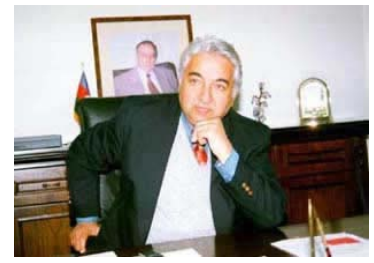

Arif PASHAYEV, Prof Eng, academician

Publications: Author of many publications, among them monographs, dealing with physical fields in solids, physics of the matter structure, modern technique of physical research.

Present position: Rector of the Azerbaijan National Academy of Aviation (Baku).

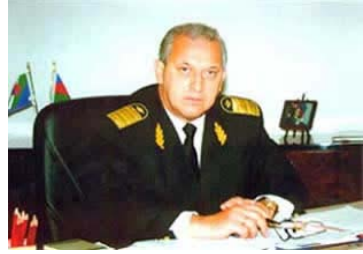

\section{Djakhangir ASKEROV}

Publications: Author of some publications in the field modeling the aviation transporting systems on the basis of modern mathematical methods and techniques.

Present position: Senior lecturer, head of "Manufacture of aviation transport" Department of the Azerbaijan National Academy of Aviation (Baku).

\section{Ramiz Ali Cabar oqlu SADIQOV, Prof Dr Habil}

Date and place of birth: March 1949, Baku Azerbaijan Republic

Education: 1971 - Azerbaijan State Pedagogy Institute, mathematician; 1985 - Institute of Cybernetics of Azerbaijan, PhD, mathematics; 1996 - Academy of Sciences, Doctor of science, Higher Attestation Committee of Russian Federation, Moscow.

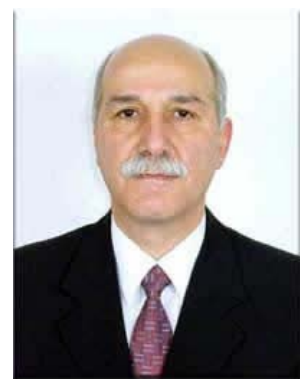

Work experience: 1998 - present Chairman and Full Professor of Department of Computer Science and Mathematical Simulation, National Aviation Academy of Azerbaijan, Baku; 1989 - 1998 Head and Associate Professor of computer center of Azerbaijan Technical University, Baku; 1977 - 1989 Head of computer center of Scientific Research Institute "Neft - Gaz - Avtomat," Baku; 1972 - 1977 Mathematician - programmer at Applied Mathematics Institute named after M.V. Keldish, Moscow; RAS

Affiliations and functions: Taught Courses: Mathematical modeling and control multiconnected systems; System theory and identification; Numerical methods; Introduction to computer science; Soft computing: fuzzy logic, neural networks, genetic algorithm, chaos and fractals.

Professional memberships: A member - academician of International Academy of Sciences, Russian section; A member - American Science Mechanical Engineering; A member - International Union of Machine Builders. Research interests: Development of mathematical models for various physical phenomena and technological processes and development numerical methods for their solving. Mathematical simulation and identification application modern technologies including Soft Computing.

Publications: The number of total published scientific works is above 250; papers on 30 scientific conferences. Present position: Chairman and Full Professor of Department of Computer Science and Mathematical Simulation, Azerbaijan National Academy of Aviation, Baku.

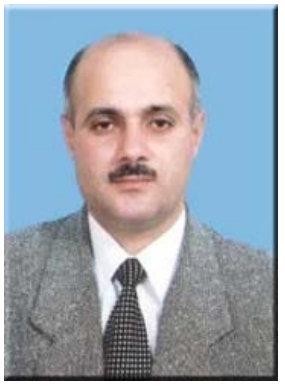

Adalat SAMEDOV, DPh

Publications: Author of many publications in the field of gas dynamics and heat transfer in gas turbines as well as cooling systems for high temperature gas turbine elements.

Present position: senior lecturer of "Design and Exploitation of Aircrafts and Aviation Engines" Department, head of "Air Transport Flight-Technical Exploitation" faculty of the Azerbaijan National Academy of Aviation (Baku).

\begin{abstract}
In contrast to methods that do not take into account multiconnectivity in a broad sense of this term, we develop mathematical models and highly effective combination (BIEM and FDM) numerical methods of calculation of stationary and quasistationary temperature field of a profile part of a blade with convective cooling (from the point of view of realization on PC). The theoretical substantiation of these methods is proved by appropriate theorems. For it, converging quadrature processes have been developed and the estimations of errors in the terms of A. Ziqmound continuity modules have been received.

For visualization of profiles the method of the least squares with automatic conjecture, device spline, smooth replenishment, and neural nets are used. Boundary conditions of heat exchange are determined from the solution of the corresponding integral equations and empirical relationships. The reliability of these methods is proved by calculation and experimental investigations heat and hydraulic characteristics of the gas turbine first stage nozzle blade.
\end{abstract}

Keywords: multiconnected systems, method of the boundary integrated equations, splints, neural networks. 


\section{Introduction}

The development of aviation gas turbine engines (AGTE) at the present stage is mainly reached by assimilation of high values of gas temperature in front of the turbine $\left(T_{\Gamma}\right)$. The activities on gas temperature increase are conducted in several directions. Assimilation of high $\left(T_{\Gamma}\right)$ in AGTE is however reached by refinement of cooling systems of turbine blades. It is especially necessary to note, that with $T_{\Gamma}$ increase the requirement to accuracy of results will increase. In other words, at allowed values of AGTE metal temperature $T_{\text {lim }}=(1100 \ldots 1300 \mathrm{~K})$, the absolute error of temperature calculation should be in limits $(20-30 \mathrm{~K})$, that is no more than $2-3 \%$.

This is difficult to achieve (multiconnected fields with various cooling channels, variables in time and coordinates boundary conditions). Such problem solving requires application of modern and perfect mathematical device.

\section{Problem formulation}

In classical statement a heat conduction differential equation in common case for non-stationary process with distribution of heat in multi-dimensional area (Fourier-Kirchhoff equation) has a kind [8]:

$$
\frac{\partial\left(\rho C_{v} T\right)}{\partial t}=\operatorname{div}(\lambda \operatorname{grad} \mathrm{T})+q_{v},
$$

where $\rho, c_{v}$ and $\lambda$ - accordingly material density, thermal capacity, and heat conduction; $q_{v}$-internal source or drain of heat, and $T$ - is required temperature.

Research has established that the temperature condition of the blade profile part with radial cooling channels can be determined as two-dimensional [12]. Besides, if to suppose constancy of physical properties and absence of internal sources (drains) of heat, then the temperature field under fixed conditions will depend only on the skew shape and on the temperature distribution on the skew boundaries. In this case, equation (1) will look like:

$$
\Delta T=\frac{\partial^{2} T}{\partial x^{2}}+\frac{\partial^{2} T}{\partial y^{2}}=0 .
$$

When determining particular temperature fields in gas turbine elements are used boundary conditions of the third kind, describing heat exchange between the skew field and the environment (on the basis of a hypothesis of a Newton-Riemann). In that case, these boundary conditions will be recorded as follows:

$$
\alpha_{0}\left(T_{0}-T_{\gamma_{0}}\right)=\lambda \frac{\partial T_{\gamma_{0}}}{\partial n} .
$$

This following equation characterizes the quantity of heat transmitted by convection from gas to unit of a surface of a blade and assigned by heat conduction in a skew field of a blade.

$$
-\lambda \frac{\partial T_{\gamma_{i}}}{\partial n}=\alpha_{i}\left(T_{\gamma_{i}}-T_{i}\right)
$$

Equation (4) characterizes the heat quantity assigned by convection of the cooler, which is transmitted by heat conduction of the blade material to the surface of cooling channels: where $T_{0}$ is the temperature of environment at $i=0 ; T_{i}$ is the temperature of the environment at $i=\overline{1, M}$ (temperature of the cooler), where $M$ is the quantity of outlines; $T_{\gamma_{0}}$ is the temperature on an outline $\gamma_{i}$ at $i=0$ (outside outline of blade); $T_{\gamma_{i}}$ is the temperature on an $\gamma_{i}$ at $i=\overline{1, M}$ (outline of cooling channels); $\alpha_{0}$ is the heat transfer factor from gas to a surface of a blade (at $i=0$ ); $\alpha_{i}$ is the heat transfer factor from a blade to the cooling air at $i=\overline{1, M} ; \lambda$ is the thermal conductivity of the material of a blade; and $n$ is the external normal on an outline of researched area.

\section{Problem solution}

At present for the solution of this boundary problem (2)-(4) four numerical methods are used: Methods of Finite Differences (MFD), Finite Element Method (FEM), probabilistic method (Monte-Carlo method), and Boundary Integral Equations Method (BIEM) (or its discrete analog - Boundary Element Method (BEM)).

Let us consider BIEM application for the solution of problem (2)-(4).

2.1. The function $T=T(x, y)$, continuous with the derivatives up to the second order, satisfying the Laplace equation in considered area, including and its outline $\Gamma=\bigcup_{i=0}^{M} \gamma_{i}$, is harmonic. Consequence of the Grin integral formula for the researched harmonic function $T=T(x, y)$ is the ratio:

$$
T(x, y)=\frac{1}{2 \pi} \int_{\Gamma}\left[T_{\Gamma} \frac{\partial(\ln R)}{\partial n}-\ln R \frac{\partial T_{\Gamma}}{\partial n}\right] d s,
$$

where $R$ is the variable at an integration of the distance between point $K(x, y)$ and "running" on the outline $k$ is the point; $T_{\Gamma}$ is the temperature on the outline $\Gamma$. The temperature value in some point $k$ lying on the boundary is determined (as limiting at approach of point $K(x, y)$ to the boundary)

$$
T_{k}=\frac{1}{2 \pi}\left[\int_{\Gamma} T_{\Gamma} \frac{\partial\left(\ln R_{k}\right)}{\partial n} d s-\int_{\Gamma} \frac{\partial T_{\Gamma}}{\partial n} \ln R_{k} d s\right] .
$$

With allowance of the boundary conditions (2)(3), after collecting terms of terms and input of new factors, the ratio (6) can be presented as a linear algebraic equation, computed for the point $R$ :

$$
\begin{aligned}
& \varphi_{k 1} T_{\gamma_{01}}+\varphi_{k 2} T_{\gamma_{02}}+\ldots+\varphi_{k n} T_{\gamma_{0 m}}- \\
& -\varphi_{k \gamma_{0}} T_{0}-\varphi_{k \gamma_{i}} T_{i}-2 \pi T_{k}=0,
\end{aligned}
$$

where $n$ is the quantity of sites of a partition of an outside outline of a blade $\ell_{\gamma_{0}}\left(\ell_{\gamma_{i}}\right.$ on $\left.i=0\right)$ on small sections $\Delta S_{0}\left(\Delta S_{i}\right.$ at $\left.i=0\right), m$ is the quantity of sites of a partition of 
outside outlines of all cooling channels $\ell_{\gamma_{i}}(i=1, M)$ on small sections $\Delta S_{i}$.

Let us note, that unknowns in the equation (7) except the unknown of true value $T_{k}$ in the $k$ point are also mean on sections of the outlines partition $\Delta S_{0}$ and $\Delta S_{i}$ temperatures $T_{\gamma_{01}}, T_{\gamma_{02}}, \ldots, T_{\gamma_{0 m}}$ and $T_{\gamma_{i 1}}, T_{\gamma_{i 2}}, \ldots, T_{\gamma_{i m}}$ (total number $n+m$ ).

From a ratio (7), we shall receive the required temperature for any point, using the formula (5):

$$
\begin{aligned}
& T(x, y)=\frac{1}{2 \pi}\left[\varphi_{k 1} T_{\gamma_{01}}+\varphi_{k 2} T_{\gamma_{02}}+\ldots+\varphi_{k n} T_{\gamma_{0 n}}+\right. \\
& \left.+\ldots+\varphi_{k m} T_{\gamma_{i m}}-\varphi_{k \gamma_{0}} T_{c p_{0}}-\varphi_{k \gamma_{i}} T_{c p_{i}}\right]
\end{aligned}
$$

where

$$
\begin{gathered}
\varphi_{\mathrm{k} 1}=\int_{\Delta \mathrm{S}_{01}} \frac{\partial\left(\ell \mathrm{nR}_{\mathrm{k}}\right)}{\partial \mathrm{n}} \mathrm{ds}-\frac{\alpha_{01}}{\lambda_{1}} \int_{\Delta \mathrm{S}_{01}} \ell \mathrm{nR}_{\mathrm{k}} \mathrm{ds} \\
\varphi_{\mathrm{kn}}=\int_{\Delta \mathrm{S}_{0 \mathrm{~m}}} \frac{\partial\left(\ell \mathrm{nR}_{\mathrm{k}}\right)}{\partial \mathrm{n}} \mathrm{ds}-\frac{\alpha_{0 \mathrm{~m}}}{\lambda_{\mathrm{m}}} \int_{\Delta \mathrm{S}_{0 \mathrm{~m}}} \ell \mathrm{nR}_{\mathrm{k}} \mathrm{ds} \\
\varphi_{\mathrm{k} \gamma_{0}}=\frac{\alpha_{01}}{\lambda_{1}} \int_{\Delta \mathrm{S}_{01}} \ell \mathrm{nR}_{\mathrm{k}} \mathrm{ds}+\ldots+\frac{\alpha_{0 \mathrm{n}}}{\lambda_{\mathrm{n}}} \int_{\Delta \mathrm{Sn}} \ell \mathrm{nR}_{\mathrm{k}} \mathrm{ds} \\
\varphi_{\mathrm{k} \gamma_{\mathrm{ii}}}=\frac{\alpha_{01}}{\lambda_{1}} \int_{\Delta \mathrm{S}_{\mathrm{il}}} \ell \mathrm{nR}_{\mathrm{k}} \mathrm{ds}+\ldots+\frac{\alpha_{\mathrm{im}}}{\lambda_{\mathrm{m}}} \int_{\Delta \mathrm{S}_{\mathrm{im}}} \ell \mathrm{nR}_{\mathrm{k}} \mathrm{ds} .
\end{gathered}
$$

In activities the discretization of aniline $\Gamma=\bigcup_{i=0}^{M} \gamma_{i}$ by a many discrete point and integrals that are included in the equations as logarithmic potentials, was calculated approximately with the following ratios [12]:

$$
\begin{aligned}
& \int_{\Delta S_{\gamma_{i}}} \frac{\partial\left(\ln R_{k}\right)}{\partial n} d s \approx \frac{\partial\left(\ell n R_{k}\right)}{\partial n} \Delta S_{\gamma_{i}}, \\
& \int_{\Delta S_{\gamma_{i}}} \ell n R_{k} d s \approx \ell n R_{k} \Delta S_{\gamma_{i}},
\end{aligned}
$$

(where $\Delta S_{\gamma_{i}} \in L=\bigcup_{i=0}^{M} l_{i} ; \quad l_{i}=\int_{\gamma_{i}} d s$ )

2.2. In contrast to L.V. Arseniev et al., we offer to decide the given boundary value problem (2)-(4) as follows [13]. We locate the distribution of temperature $T=T(x, y)$ as follows:

$$
T(x, y)=\int_{\Gamma} \rho \ln R^{-1} d s,
$$

where $\Gamma=\bigcup_{i=0}^{M} \gamma_{i}$-smooth closed Jordan curve; $M$-quantity of cooled channels; $\rho=\bigcup_{i=0}^{M} \rho_{i}$ - density of a logarithmic potential uniformly distributed on $\gamma_{i} \quad S=\bigcup_{i=0}^{M} s_{i}$.

Thus curve $\Gamma=\bigcup_{i=0}^{M} \gamma_{i}$ are positively oriented and are given in a parametric kind: $x=x(s) ; y=y(s)$; $s \in[0, L] ; L=\int_{\Gamma} d s$.

Using BIEM and expression (11) we shall put problem (2)-(4) to the following system of boundary integral equations:

$$
\begin{aligned}
& \rho(s)-\frac{1}{2 \pi} \int_{\Gamma}(\rho(s)-\rho(\xi)) \frac{\partial}{\partial n} \ln R(s, \xi) d \xi= \\
& =\frac{\alpha_{i}}{2 \pi \lambda}\left(T-\int_{\Gamma} \rho(s) \ell n R^{-1} d s\right),
\end{aligned}
$$

where

$$
R(s, \xi)=\left((x(s)-x(\xi))^{2}+(y(s)-y(\xi))^{2}\right)^{1 / 2} .
$$

For the singular integral operator's evaluation, which are included in (12) the discrete operators of the logarithmic potential with simple and double layer are investigated. Their connection and the evaluations in modules term of the continuity (evaluation such as assessments by A. Zigmound are obtained) are shown:

Theorem (main)

Let

$$
\int_{0} \frac{\omega_{\xi}(x)}{x}<+\infty .
$$

And let the equation (12) have the solution $\mathrm{f}^{*} \in \mathrm{C}_{\Gamma}$ (the set of continuous functions on $\Gamma$ ). Then $\exists \mathrm{N}_{0} \in \mathrm{N}=\{1,2 \ldots\}$ such that the discrete system $\forall \mathrm{N}>\mathrm{N}_{0}$, obtained from (12) by using the discrete double layer potential operator (its properties has been studied), has unique solution $\left\{\hat{f}_{j_{k}}^{(N)}\right\}, k=\overline{1, m_{j}} ; j=\overline{1, n}$;

$$
\begin{aligned}
& \left|\mathrm{f}_{\mathrm{jk}}^{*}-\widehat{\mathrm{f}}_{\mathrm{jk}}^{(\mathrm{N})}\right| \leq \mathrm{C}(\Gamma)\left(\int_{0}^{\varepsilon_{\mathrm{N}}} \frac{\omega_{\xi}(\mathrm{x}) \omega_{\mathrm{f}^{*}}(\mathrm{x})}{\mathrm{x}} \mathrm{dx}+\right. \\
& +\varepsilon \int_{\varepsilon_{\mathrm{N}}}^{\mathrm{L} / 2} \frac{\omega_{\xi}(\mathrm{x}) \omega_{\mathrm{f}^{*}}(\mathrm{x})}{\mathrm{x}} \mathrm{dx}+\omega_{\mathrm{f}^{*}}\left(\left\|\tau_{\mathrm{N}}\right\|\right) \int_{0}^{\mathrm{L} / 2} \frac{\omega_{\mathrm{f}^{*}}(\mathrm{x})}{\mathrm{x}} \mathrm{dx}+ \\
& \left.+\left\|\tau_{\mathrm{N}}\right\| \int_{\varepsilon_{\mathrm{N}}}^{\mathrm{L} / 2} \frac{\omega_{\mathrm{f}^{*}}(\mathrm{x})}{\mathrm{x}} \mathrm{dx}\right),
\end{aligned}
$$

where $C(\Gamma)$ is constant, depending only on $\left\|\tau_{N}\right\|_{N=1}^{\infty}$ is the sequence of partitions of $\Gamma ;\left\{\varepsilon_{N}\right\}_{N=1}^{\infty}$ is the sequence of positive numbers such that the pair $\left(\left\|\tau_{N}\right\|, \varepsilon_{N}\right)$, satisfies the condition $2 \leq \varepsilon\|\tau\|^{-1} \leq \mathrm{p}$.

Let $\delta \in(0, d / 2)$, where $\mathrm{d}$ is diameter $\Gamma$, and the splitting $\tau$ is that, which is satisfied the condition

$$
p^{\prime} \geq \delta /\|\tau\| \geq 2
$$

Then for all $\psi \in C_{\Gamma} \quad\left(C_{\Gamma}\right.$ - space of all functions continuous on $\Gamma)$ and $z \in \Gamma,(z=x+i y)$

$$
\begin{aligned}
& \left|\left(I_{\tau, \delta} f\right)(z)-\bar{f}(z)\right| \leq C(\Gamma) \\
& \left(\|f\|_{C} \delta \ln \frac{2 d}{\delta}+\omega_{f}(\|\tau\|)+\|\tau\| \ln \frac{2 d}{\delta}+\|f\|_{C} \omega_{Z}(\|\tau\|)\right) ; \\
& \left|\left(L_{\Omega, \Gamma} f\right)(z)-\tilde{f}(z)\right| \leq\left(C(\Gamma) \int_{0}^{\Gamma} \frac{\omega_{f}(x) \omega_{l}(x)}{x^{2}} d x+\right. \\
& \left.+\omega_{f}(\|\tau\|) \int_{\Delta}^{d} \frac{\omega_{l}(x)}{x} d x+\|\tau\| \int_{\Delta}^{d} \frac{\omega_{f}(x)}{x^{2}} d x\right)
\end{aligned},
$$


where

$$
\begin{aligned}
& \left(L_{\tau, \varepsilon} f\right)(z)=\sum_{z_{m, e \in \tau}(z)}\left(\frac{f\left(z_{k, e+1}\right)+f\left(z_{k, e}\right)}{2}-f(z)\right) \\
& \frac{\left(y_{k, e+1}-y_{k, e}\right)\left(x_{k, e}-x\right)-\left(x_{k, e+1}-x_{k, e}\right)\left(y_{k, e}-y\right)}{\left|z-z_{k, e}\right|^{2}}+\pi f(z) .
\end{aligned}
$$

$\left(L_{\tau, \varepsilon} f\right)(z)$ is the two-parameter quadrate formula (depending on $\tau$ and $\delta$ parameters) for logarithmic double layer potential; $\tilde{f}(z)$ is the double layer logarithmic potential operator; $C(\Gamma)$ is the constant, dependent only from a curve $\Gamma ; \omega_{f}(x)$ is a module of a continuity of functions $\mathrm{f}$;

$$
\begin{aligned}
& \left(I_{\tau, \varepsilon} f\right)(z)=\sum_{z_{m, e \epsilon \tau(z)}} \frac{f\left(z_{k, j+1}\right)+f\left(z_{k, j}\right)}{2} . \\
& \cdot \ln \frac{1}{\left|z_{k, j}-z\right|}\left|z_{k, j+1}-z_{k, j}\right|
\end{aligned}
$$

$\left(I_{\tau, \varepsilon} f\right)(z)$ is a two-parameter quadrate formula (depending on $\tau$ and $\delta$ parameters) for logarithmic potential simple layer; $\dddot{f}(z)$ is a simple layer logarithmic potential operator;

$$
\begin{aligned}
& z_{k, e} \in \tau, z_{k, e}=x_{k, e}+i y_{k, e} \\
& \tau(z)=\left\{z_{k, e}\left|z_{k, e}-z\right|>\varepsilon\right\} \\
& \tau_{k}=\left\{z_{k, 1}, \ldots, z_{k, m_{k}}\right\}, z_{k, 1} \leq z_{k, 2} \leq \ldots \leq z_{k, m_{k}} \\
& \|\tau\|=\max _{j=1, m_{k}}\left|z_{k, j+1}-z_{k, j}\right|
\end{aligned}
$$

Thus are developed effective from the point of view of realization on computers the numerical methods basing on constructed two-parametric quadrate processes for the discrete operators logarithmic potential of the double and simple layer. Their systematic errors are estimated; the methods quadratures mathematically are proved for the approximate solution Fredholm I and II boundary integral equations using Tikhonov regularization and are proved appropriate theorems [9].

2.3. The given calculating technique of the blade temperature field can be applied also to blades with the plug-in deflector. On consideration blades with deflectors in addition to boundary condition of the III kind adjoin also interfaces conditions between segments of the outline partition as equalities of temperatures and heat flows

$$
\begin{aligned}
& T_{v}(x, y)=T_{v+1}(x, y), \\
& \frac{\partial T_{v}(x, y)}{\partial n}=\frac{\partial T_{v+1}(x, y)}{\partial n},
\end{aligned}
$$

where $v$ is the number of segments of the outline partition of the blade cross-section; and $x, y$ is a coordinates of segments. At finding of cooler $\mathrm{T}$ best values, is necessary to solve the inverse problem of heat conduction. For it is necessary at first to find solution of the heat conduction direct problem with boundary condition of the III kind from a gas leg and boundary conditions I kinds from a cooling air leg

$$
\left.T_{v}(x, y)\right|_{\gamma_{0}}=T_{i_{0}}
$$

where $T_{i_{0}}$ is the unknown optimum temperature of a wall of a blade from a leg of a cooling air.

2.4. The developed technique for the numerical solution of stationary task of the heat conduction in cooled blades can be distributed also to quasistationary case.

Let us consider a third boundary-value problem for the heat conduction quasilines equation:

$$
\begin{aligned}
& \frac{\partial}{\partial x}\left(\lambda(T) \frac{\partial T}{\partial x}\right)+\frac{\partial}{\partial y}\left(\lambda(T) \frac{\partial T}{\partial y}\right)=0 \\
& \alpha_{i}\left(T_{c i}-T_{\gamma i}\right)-\lambda(T) \frac{\partial T_{\gamma i}}{\partial n}=0
\end{aligned}
$$

For linearization of tasks (16) - (17) we shall use the Kirchoff permutation:

$$
A=\int_{0}^{T} \lambda(\xi) d \xi
$$

Then equation (16) is transformed into the following Laplace equation:

$$
\frac{\partial^{2} A}{\partial x^{2}}+\frac{\partial^{2} A}{\partial y^{2}}=0
$$

For preserving convection additives in boundary-value condition (17), we shall accept in initial approximation $\lambda(T)=\lambda_{c}$. Then from (18) we have

$$
T=A / \lambda c
$$

And the regional condition (17) will be transformed as follows:

$$
\alpha_{i}\left(T_{c i}-A_{\gamma i} / \lambda_{c}\right)-\frac{\partial A_{\gamma i}}{\partial n}=0
$$

So, the stationary problem (19) with (21) is solved by boundary integrated equations method. If the solution $L(x, y)$ in the $(x, y)$ point of the linear third boundaryvalue problem (19), (21) for the Laplace equation substitute in (18) and after integration to solve the appropriate algebraic equation, which degree is higher than the degree of function $\lambda(T)$ with digit, we shall receive meaning of temperature $T(x, y)$ in the same point. Thus in radicals is solved the algebraic equation with non-above fourth degree

$$
a^{0} T^{4}+a^{1} T^{3}+a^{2} T^{2}+a^{3} T+a^{4}=A .
$$

This corresponds to the $\lambda(T)$ as the multinomial with degree non-above third. In the result, the temperature field will be determined on the first approximation, as the boundary condition (17) took into account constant meaning heat conduction $\lambda_{c}$ in convective thermal flows. According to it we shall designate this solution $T^{(l)}$ (accordingly $A^{(I)}$ ). For determining consequents approximations $A^{(2)}$ (accordingly $T^{(2)}$ ), the function $A(T)$ is decomposing in Taylor series in the neighborhood of $T^{(l)}$ and the linear members are left in it only. In result is received a third boundary-value problem for the Laplace equation relatively function $A^{(2)}$. The 
temperature $T^{(2)}$ is determined by the solution of the equation (20).

2.5. The multiples computing experiments with the using BIEM for calculation the temperature fields of nozzle and working blades with various amount and disposition of cooling channels, having a complex configuration, is showed, that for practical calculations in this approach, offered by us, the discretization of the integrations areas can be conducted with smaller quantity of discrete points. Thus the reactivity of the algorithms developed and accuracy of evaluations is increased. The accuracy of temperatures calculation, required consumption of the cooling air, heat flows, losses from cooling margins essentially depends on reliability of boundary conditions, included in calculation of heat exchange.

2.6. Piece-polynomial smoothing of cooled gas-turbine blade structures with automatic conjecture is considered: the method of the least squares, device spline, smooth replenishment, and neural nets are used.

2.6.1. Let the equation of the cooled blade outline segments is the third degree polynomial:

$$
y(x)=a_{0}+a_{1} x+a_{2} x^{2}+a_{3} x^{3}
$$

The equation of measurements of the output coordinate has a kind:

$$
Z_{y}=a_{0}+a_{1} x+a_{2} x^{2} a_{3} x^{3}+\delta_{y}
$$

where $\mathrm{Zy}=\left\|\mathrm{z}_{1 \mathrm{y}}, \mathrm{z}_{2 \mathrm{y}}, \ldots, \mathrm{z}_{\mathrm{ny}}\right\|^{\mathrm{T}}$ - vector of measurements of output coordinate, n-amount of the points in the consideration interval. For coefficients of polynomial (23) estimate the method of the least squares of the following kind is used

$$
\begin{aligned}
& \hat{\theta}=\left(\mathrm{X}^{\mathrm{T}} \mathrm{X}\right)^{-1}\left(\mathrm{X}^{\mathrm{T}} \mathrm{Z}_{\mathrm{y}}\right), \\
& D_{\hat{\theta}}=\left(\mathrm{X}^{\mathrm{T}} \mathrm{X}\right)^{-1} \sigma^{2},
\end{aligned}
$$

where

$$
\mathrm{X}=\left\|\begin{array}{llll}
1 & x_{1} & x_{1}^{2} & x_{1}^{3} \\
1 & x_{2} & x_{2}^{2} & x_{2}^{3} \\
1 & x_{3} & x_{3}^{2} & x_{3}^{3} \\
\cdots & \cdots & \cdots & \cdots \\
1 & x_{n} & x_{n}^{2} & x_{n}^{3}
\end{array}\right\| \text { - structural matrix; }
$$

$D_{\widehat{\theta}}$ - dispersion matrix of errors; $\hat{\theta}=\left\|\mathrm{a}_{0}, \mathrm{a}_{1}, \mathrm{a}_{2}, \mathrm{a}_{3}\right\|^{\mathrm{T}}$ vector of estimated coefficients.

Estimations of coefficients for the first segment is received with using formula (25). Beginning with second segment, the $\theta$ vectors components is calculated on experimental data from_this segment, but with the account of parameters found on the previous segments. Thus, each subsequent segment of the blade cross-section outline we shall choose with overlapping. Thus, it is expedient to use the following linear connections between the estimated parameters of the previous segment $\hat{\theta}_{N_{1}}$ and required $\hat{\theta}_{N}$ for N-th segment:
$\mathrm{A} \theta_{N}=\mathrm{V}$,

$$
\mathrm{A}=\left[\begin{array}{cccc}
1 & x_{e} & x_{e}^{2} & x_{e}^{3} \\
0 & 1 & 2 x_{e} & 3 x_{e}^{2} \\
0 & 0 & 2 & 6 x_{e}
\end{array}\right]
$$

$$
\mathrm{V}=\left[\begin{array}{l}
\hat{a}_{0 N-1}+\widehat{a}_{1 N-1} x_{e}+\hat{a}_{2 N-1} x_{e}^{2}+\hat{a}_{3 N-1} x_{e}^{3} \\
\hat{a}_{1 N-1}+\widehat{a}_{2 N-1} x_{e}+3 \widehat{a}_{3 N-1} x_{e}^{2} \\
2 \widehat{a}_{2 N-1}+6 \hat{a}_{3 N-1} x_{e}
\end{array}\right],
$$

$\mathrm{e}=(\mathrm{N}-1)(\mathrm{n}-\mathrm{L})$; L- number points of overlapping.

The expressions (27)-(29) describe communications, which provide joining of segments of interpolation on function with first and second degrees.

Taking into account the accuracy of measurements, the problem of defining unknown coefficients of the model in this case can be formulated as a problem conditional extremum: minimization of the quadratic form $\left(Z_{\mathrm{y}}-\mathrm{X} \theta\right)^{\mathrm{T}} \sigma^{2} \mathrm{I}\left(\mathrm{Z}_{\mathrm{y}}-\theta\right)$ under the limiting condition (27). Here $I$ is a individual matrix.

For the solution of such problems, usually are using the method of Lagrange uncertain multipliers. In result, we shall write down the following expressions for estimation vector of coefficients at linear connections presence (27):

$$
\begin{aligned}
& \tilde{\theta}^{\mathrm{T}}=\hat{\theta}^{\mathrm{T}}+\left(\mathrm{V}^{\mathrm{T}}-\hat{\theta}^{\mathrm{T}} \mathrm{A}^{\mathrm{T}}\right)\left[\mathrm{A}\left(\mathrm{X}^{\mathrm{T}} \mathrm{X}\right)^{-1} \mathrm{~A}^{\mathrm{T}}\right]^{-1} \mathrm{~A}\left(\mathrm{X}^{\mathrm{T}} \mathrm{X}\right)^{-1}, \\
& D_{\tilde{\theta}}=D_{\hat{\theta}}-\left(\mathrm{X}^{\mathrm{T}} \mathrm{X}\right)^{-1} \mathrm{~A}^{\mathrm{T}}\left[\mathrm{A}\left(\mathrm{X}^{\mathrm{T}} \mathrm{X}\right)^{-1} \mathrm{~A}^{\mathrm{T}}\right]^{-1} \mathrm{~A}\left(\mathrm{X}^{\mathrm{T}} \mathrm{X}\right)^{-1} \sigma^{2},
\end{aligned}
$$

Substituting matrixes $A$ and $X$ and vectors $Z_{\mathrm{y}}$ and $\mathrm{V}$ in expressions (25), (26), (30), and (31), we receive estimations of the vector of coefficients for segment of the cooled blade section with number $\mathrm{N}$ and also the dispersing matrix of errors.

As a result of consecutive application of the described procedure and with using of experimental data, we shall receive peace-polynomial interpolation of the researched segments with automatic conjecture.

Research showed that optimum overlapping in most cases is the $50 \%$-overlapping.

2.6.2. Besides peace-polynomial regression exist interpolation splines which represent polynomial (low odd degrees - third, fifth), subordinated to the condition of function and derivatives (first and second in case of cubic spline) continuity in common points of the next segments. If the equation of the cooled gas-turbine blades profile is described cubic spline submitted in obvious polynomial kind (23), the coefficients $a_{0}, a_{1}, a_{2}, a_{3}$ determining $\mathrm{j}$-th spline, i.e. line connecting the points $Z_{j}=\left(x_{j}, y_{j}\right)$ and $\mathrm{Z}_{\mathrm{j}+1}=\left(\mathrm{x}_{\mathrm{j}+1}, \mathrm{y}_{\mathrm{j}+1}\right)$, are calculating as follows:

$a_{0}=z_{j}$

$a_{1}=z_{j}^{\prime} ;$

$a_{2}=z_{j}^{\prime \prime} / 2=3\left(z_{j+1}-z_{j}\right) h_{j+1}^{-2}-2 z_{j}^{\prime} h_{j+1}^{-1}-z_{j+1}^{\prime} h_{j+1}^{-1} ;$

$a_{3}=z_{j}^{\prime \prime \prime} / 6=2\left(z_{j}-z_{j+1}\right) h_{j+1}^{-3}+z_{j}^{\prime} h_{j+1}^{-2}+z_{j+1}^{\prime} h_{j+1}^{-2}$;

where $h_{j+1}=\left|z_{j+1}-z_{j}\right|, j=\overline{1, N-1}$. 
2.6.3. Let us consider other way smooth replenishment of the cooled gas-turbine blade profile on the precisely measured meaning of coordinates in final system of discrete points, distinguishing from spline-function method and also from the point of view of effective realization on computers.

Let equation cooled blades profile segments are described by the multinomial of the third degree of the type (23). By taking advantage the smooth replenishment method (conditions of function smooth and first derivative are carried out) we shall define its coefficients:

$$
\begin{aligned}
& a_{0}=z_{j} ; \\
& a_{1}=\left(z_{j+1}-z_{j}\right) h_{j+1}^{-1} ; \\
& a_{2}=-\left(\left(z_{j+2}-z_{j+1}\right) h_{j+2}^{-1}+\left(z_{j+1}-z_{j}\right) h_{j+1}^{-1}\right) h_{j+1}^{-1} ; \\
& a_{3}=\left(\left(z_{j+2}-z_{j+1}\right) h_{j+2}^{-1}-\left(z_{j+1}-z_{j}\right) h_{j+1}^{-1}\right) h_{j+1}^{-2} ; \\
& j=1, N-1-S, S=1 .
\end{aligned}
$$

If it's required carry out conditions of function smooth first and second derivatives, i.e. corresponding to cubic splines smooth, we shall deal with the multinomial of the fifth degree (degree of the multinomial is equal $2 \mathrm{~S}+1$, i.e. $\mathrm{S}=2$ ).

The advantage of such approach (smooth replenishment) is that it's not necessary to solve system of the linear algebraic equations, as in case of the spline application, though the degree of the multinomial is higher 2.

2.6.4. A new approach of mathematical models' parameters identification is considered. This approach is based on Neural Networks (Soft Computing) [1, 12, 5]. Let us consider the regression equations:

$$
\begin{aligned}
& Y_{i}=\sum_{j=1}^{n} a_{i j} x_{j} ; i=\overline{1, m} \\
& Y_{i}=\sum_{r, s} a_{r s} x_{1}^{r} x_{2}^{s} ; r=\overline{0, l} ; s=\overline{0, l} ; r+s \leq l,
\end{aligned}
$$

where $a_{r s}$ are the required parameters (regression coefficients).
The problem is put definition of values $a_{i j}$ and $a_{r s}$ parameters of equations (34) and (35) based on the statistical experimental data, i.e. input $x_{j}$ and $x_{1}, x_{2}$, output coordinates $Y$ of the model.

Neural Network (NN) consists from connected between their neurons sets. At using $\mathrm{NN}$ for the solving (34) and (35) input signals of the network are accordingly values of variables $X=\left(x_{1}, x_{2}, \ldots, x_{n}\right), X=\left(x_{1}, x_{2}\right)$ and output $Y$.

At the solving of the identification problem of parameters $a_{i j}$ and $a_{r s}$ for the equations (34) and (35) with using $\mathrm{NN}$, the basic problem is training the last.

We allow, there are statistical data from experiments. On the basis of these input and output data we making training pairs $(X, T)$ for network training. For construction of the model process on input of $\mathrm{NN}$ input signals $X$ move and outputs are compared with reference output signals $T$.

After comparison, the deviation value is calculating by formula

$$
E=\frac{1}{2} \sum_{j=1}^{k}\left(Y_{j}-T_{j}\right)^{2} .
$$

If for all training pairs, deviation value $E$ less given then training (correction) parameters of a network comes to end (Fig 1). In opposite case it continues until value $E$ will not reach minimum.

Correction of network parameters for left and right part is carried out as follows:

$$
a_{r s}^{H}=a_{r s}^{c}+\gamma \frac{\partial E}{\partial a_{r s}},
$$

where $a_{r s}^{c}, a_{r s}^{H}$ are the old and new values of $\mathrm{NN}$ parameters and $\gamma$ is training speed.

The structure of NN for identifying the parameters of the equation (34) is given on Fig 2.

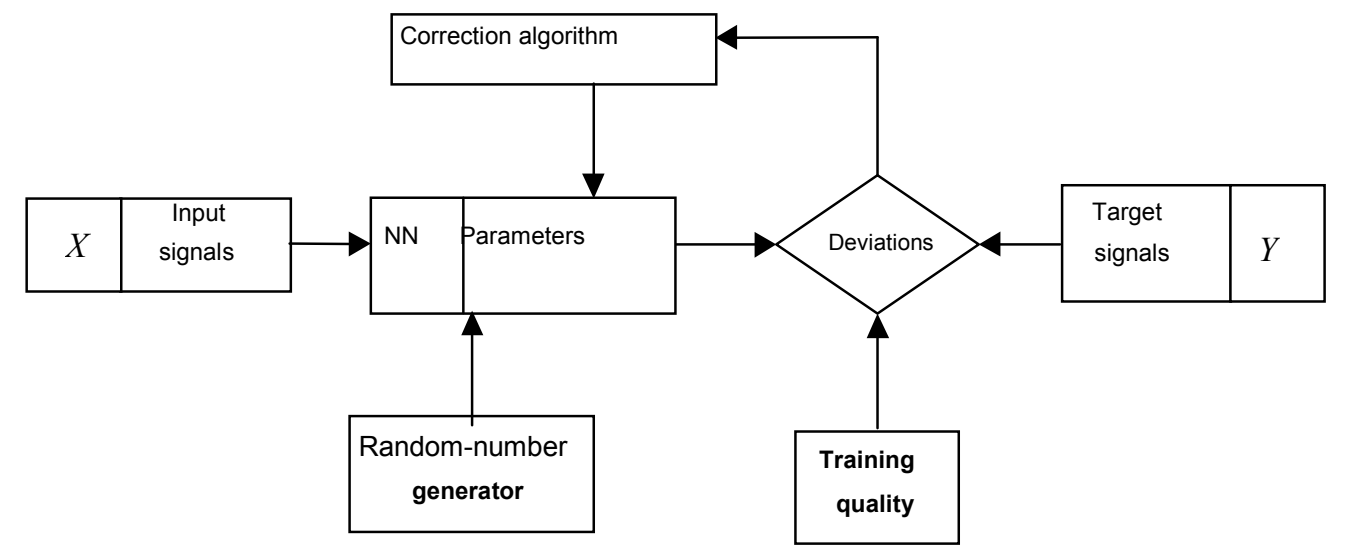

Fig 1. System for network-parameter (weights, threshold) training (with feedback) 


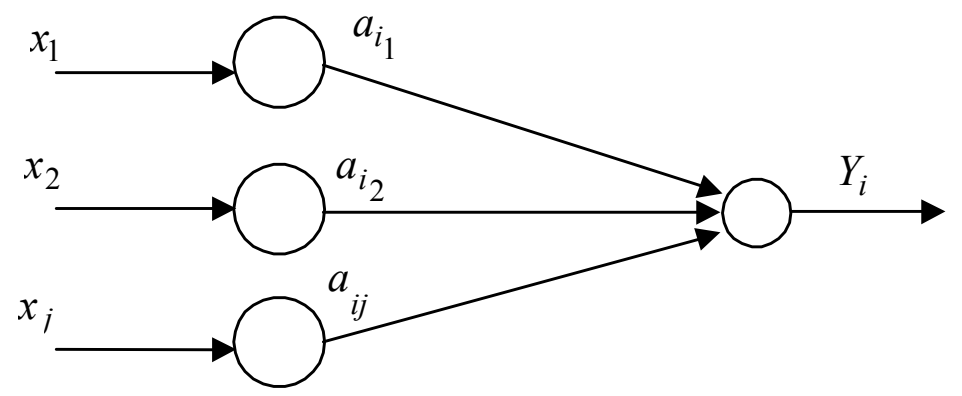

Fig 2. Neural network structure for multiple linear regression equation

2.7. For determining of the temperature fields of AGTE elements, the problem of gas flow distribution on blades' profile of the turbine cascade is considered. The solution is based on the numerical realization of the Fredholm boundary integrated equation II kind.

2.7.1. On the basis of the theory of the potential flow of cascades, distribution of speed along the profile contour can be found by solving of the following integrated equation [9]:

$\varphi\left(x_{k}, y_{k}\right)=V_{\infty}\left(x_{k} \cos \alpha_{\infty}+y_{k} \sin \alpha_{\infty}\right) \pm \frac{1}{2 \pi} \Gamma \theta_{B} \mp \frac{1}{2 \pi} \int_{S+} \varphi(S) d \theta,(36$

where $\varphi\left(x_{k}, y_{k}\right)$ is the value of speeds potential; $V_{\infty}$ is the gas speed vectors mean on the flowing; $\alpha_{\infty}$ - is the angle between the vector $V_{\infty}$ and the profile cascade axis; $\Gamma$ is the circulation of speed; and $\theta_{B}$ is the angle that corresponds to the outlet edge of the profile.

For the numerical solution of the integrated equation (36) the following approximating expression is received:

$$
\varphi_{j} \pm \sum_{i=1}^{n} \varphi_{i}\left(\theta_{j, i+1}-\theta_{j, i-1}\right)=V_{\infty}\left(x_{k_{j}} \cos \alpha_{\infty}+y_{k_{j}} \sin \alpha_{\infty}\right) \pm \frac{1}{2 \pi} \Gamma \theta_{j, B},
$$

where $i=2 n-1, j=2 n, n$ is the numbers of parts.

Distribution of speeds potential $\varphi$ along the profile contour received from the solution of linear algebraic equations system.

The value of the gas flow speed is determined by the derivation of speeds potential along the contour $s$, i.e. $V(s)=d \varphi / d s$.

2.7.2. Distribution of speed along the profile contour can be determined by solving the integral equation for the current function $\psi[9,3]$ :

$\psi=V_{\infty}\left(y \cos \alpha_{\infty}-x \sin \alpha_{\infty}\right) \mp \frac{1}{2 \pi} \oint_{S+} V \ln \sqrt{\operatorname{sh}^{2} \frac{\pi}{t}\left(x-x_{k}\right)+\sin ^{2} \frac{\pi}{t}\left(y-y_{k}\right)} d s$

taking it to simple algebraic type:

$\psi=\psi_{\infty} \mp \frac{1}{2 \pi} \sum_{i=1}^{n} V_{i} \ln \left\{\sqrt{\operatorname{sh}^{2}\left[\frac{2 \pi}{t}\left(x-x_{k}\right)\right]-\sin ^{2}\left[\frac{2 \pi}{t}\left(y-y_{k}\right)\right]}\right\} \Delta s_{i}$

2.8. The data of speed distribution along the profile contour are incoming for determining outer boundary heat exchange conditions.
Тhe "ЦКТИ" method is used for finding the local heat transfer coefficient $\alpha_{2}$ in this case [13].

At the thickened entrance edges characteristic of cooled gas vanes, the outer local heat exchange is described by empirical dependences offered by E.G. Roost [13]:

where

$$
N u_{x}=0,5 \cdot \operatorname{Re}_{x}^{0,5} \cdot \varepsilon_{T u},
$$

$$
\begin{aligned}
\text { at } 1,0 \%<T u<3,5 \%: \varepsilon_{T u} & =1+T u^{1,4} / 10 ; \\
\text { at } 3,5 \%<T u \quad: \varepsilon_{T u} & =1+4 T u^{0,28} / 10
\end{aligned}
$$

2.9. The problem of determining inner boundary heat exchange conditions is necessary.

For example, to calculate heat transfer in the cooling channel track of the vanes of deflector construction usually is applied criterial relationships. The mean coefficients on the inner surface of the carrier envelope at the entrance edge zone under the condition of its spray injection by the number of sprays from round holes in the nose deflector were obtained by the equation [7]:

$$
N u=C \boldsymbol{R e}^{0.98} \operatorname{Pr}^{0.43} /\left(L / b_{\text {equ }}\right),
$$

where $b_{\text {equ }}=\pi d_{0}^{2} / 2 t_{0}$ - the width of hole which is equivalent by the trans area; $d_{0}, t_{0}$-diameter and pitch of the holes in nose deflector. The Re criterion in this formula is determined by the speed of the flow from the holes at the exit in the nose deflector and the length $\mathrm{L}$ of the carrier wall in the entrance edge zone.

The empirical criterion equation earlier received [6]:

$$
\begin{aligned}
& N u=0.018\left(0.36 \bar{\delta}^{2}-0.34 \bar{\delta}+0.56-0.1 \bar{h}\right) \bar{S}_{x} . \\
& \cdot\left(G_{c} f_{k} / G_{k} f_{c}\right)^{k} \cdot \operatorname{Re}^{0.8},
\end{aligned}
$$

was used for the calculation of the mean coefficient of heat transfer at the inner surface of the vane wall in the area of the perforated deflector.

In this equation: $\bar{\delta}=\delta / d$ is the relative width of the deflector; $\bar{h}=h / d$ is the relative height of the slot channel between deflector and vane wall; $\bar{S}=S / d$ is the relative longitudinal step of perforations holes system; $d$ is the diameter of perforation; $L=0.75-0.45 \bar{\delta}$; $k=0.25+0.5 \bar{h}$. The Reynolds criterion in the formula 
(37) is defined by hydraulic diameter of cross-section channel and speed of cooler flow in the channel after the zone of deflector perforation.

2.10. At known geometry of the cooling scheme, for definition of the convective heat exchange local coefficients $\alpha_{B}$ of the cooler by the standard empirical formulas, is necessary to have income values of airflow distribution in cooling channels.

For example, for blades with deflector and with cross current, the value of the airflow $G_{B}$ for blade cooling is possible to define with the following dependence:

$$
G_{B}=\frac{\mu_{B} F_{B}}{d_{B}}\left(\frac{d_{B}}{\lambda_{B} \cdot C}\right)^{\frac{1}{n}}\left[\frac{\alpha_{\Gamma}\left(\psi_{\Gamma}-1\right) \kappa_{\phi}}{1-\frac{2 B i\left(\psi_{\Gamma}-1\right) \kappa_{\phi}}{1+\kappa_{\phi}}-\psi_{B}}\right]^{\frac{1}{n}},
$$

where $\psi_{\Gamma}, \psi_{B}$ is the gas and air temperature coefficients; $\kappa_{\phi}$ is the coefficient of the form; $d_{B}$ is the characteristic size in the formula $\operatorname{Re}_{B} ; \mu_{B}, \lambda_{B}$ is the cooler dynamic viscosity and heat conductivity coefficients; $B i$ is the Bio criterion for the blade wall; $F_{B}$ is the total area of passage for air; $C$ and $n$ is the coefficient and exponent ratio in criteria formulas for convective heat exchange $N u_{B}=C \operatorname{Re}_{B}^{n}$ for considered cooling parts.

2.11. To determine the distribution of flow in the blade cooling system, an equivalent hydraulic scheme is built.

The construction of the equivalent hydraulic tract circuit of the vane cooling is connected with the description of the cooled vane design. The whole passage of coolant flow is divided in some definite interconnected sections, the so-called typical elements, and every one has the possibility of identical definition of hydraulic resistance. The points of connection of typical elements are changed by node points, in which the streams, mergion or division of cooler flows is taking places proposal without pressure change. All the typical elements and node points are connected in the same sequence and order as the tract sites of the cooled vane.

To describe the coolant flow at every inner node the $1^{\text {st }}$ low by Kirchhoff is used:

$$
f_{l}=\sum_{j=1}^{m} G_{i j}=\sum_{j=1}^{m} \operatorname{sign}\left(\Delta p_{i j}\right) k_{i j} \sqrt{\Delta p_{i j}} ; \quad i=1,2,3 \ldots . n
$$

where $G_{i j}$ is the discharge of coolant on the element, $i-j, m$ are the e number of typical elements connected to $i$ node of the circuit, $n$ is the number of inner nodes of hydraulic circuit, $\Delta p_{i j}$ - losses of total pressure of the coolant on element $i-j$. In this formula the coefficient of hydraulic conductivity of the circuit element $(i-j)$ is defined as:

$$
k_{i j}=\sqrt{2 f_{i j}^{2} \cdot p_{i j} / \xi_{i j}},
$$

where $f_{i j}, p_{i j}, \xi_{i j}$ are the mean area of the cross-section passage of elements $(i-j)$, density of coolant flow in the element, and coefficient of hydraulic resistance of this element. The system of nonlinear algebraic equations (38) is solved by the Zeidel method with acceleration, taken from:

$$
p_{i}^{k+1}=p_{i}^{k}-f_{i}^{k} /(\partial f / \partial p)^{k},
$$

where $k$ is the iteration number, $p_{i}^{k}$ is the coolant pressure in $i$ node of the hydraulic circuit. The coefficients of hydraulic resistance $\xi_{i j}$ used in (39) are defined by analytical dependencies, which are in the literature available at present [7].

For example, to calculate a part of the cooling tract that includes the area of deflector perforation coefficients of hydraulic resistance in spray [2]:

$$
\xi_{c \Sigma}=\frac{\left(G_{r} / G_{k}\right)^{m}}{2\left(f_{c} / f_{k}\right)-0.1}, \quad m=\frac{1}{2\left(f_{c} / f_{k}\right)-0.23}
$$

and in general channel:

$$
\xi_{c \Sigma}=1.1\left(G_{r} / G_{k}\right)^{n}, \quad n=0.381 \ln \left(f_{c} / f_{k}\right)-0.3
$$

In these formulas, $G_{r}, G_{k}$ are cooling air consumption in the spray stream through the perforation deflector holes and slot channel between the deflector and vanes wall, and $f_{r}, f_{k}$ - the flow areas.

\section{Results}

The developed techniques of profiling, calculation of temperature fields and parameters of the cooler in cooling systems are approved at research of the gas turbine I st stage nozzle blades thermal condition. Thus the following geometrical and regime parameters of the stage are used: step of the cascade $-t=41.5 \mathrm{Mm}$, inlet gas speed to cascade $-V_{1}=156 \mathrm{M} / \mathrm{s}$, outlet gas speed from cascade $-V_{2}=512 \mathrm{M} / \mathrm{s}$, inlet gas speed vector angle $-\alpha_{1}=0.7^{0}$, gas flow temperature and pressure: on the entrance to the stage $-T_{2}^{*}=1333 \mathrm{~K}$, $p_{2}^{*}=1.2095 \cdot 10^{6} \mathrm{~Pa}$, on the exit from stage $T_{21}=1005 \mathrm{~K}, p_{21}=0.75 \cdot 10^{6} \mathrm{~Pa}$; relative gas speed on the exit from the cascade $-\lambda_{\text {lad }}=0.891$.

The geometrical model of the nozzle blades (Fig 3 ), diagrams of speed distributions $V$ and convective heat exchange local coefficients of gas $\alpha_{2}$ along profile contour (Fig 4) are received.

The geometrical model (Fig 5) and the cooling tract equivalent hydraulic scheme (Fig 6) are developed. Cooler basics parameters in the cooling system and temperature field of blade cross section (Fig 7) are determined. 


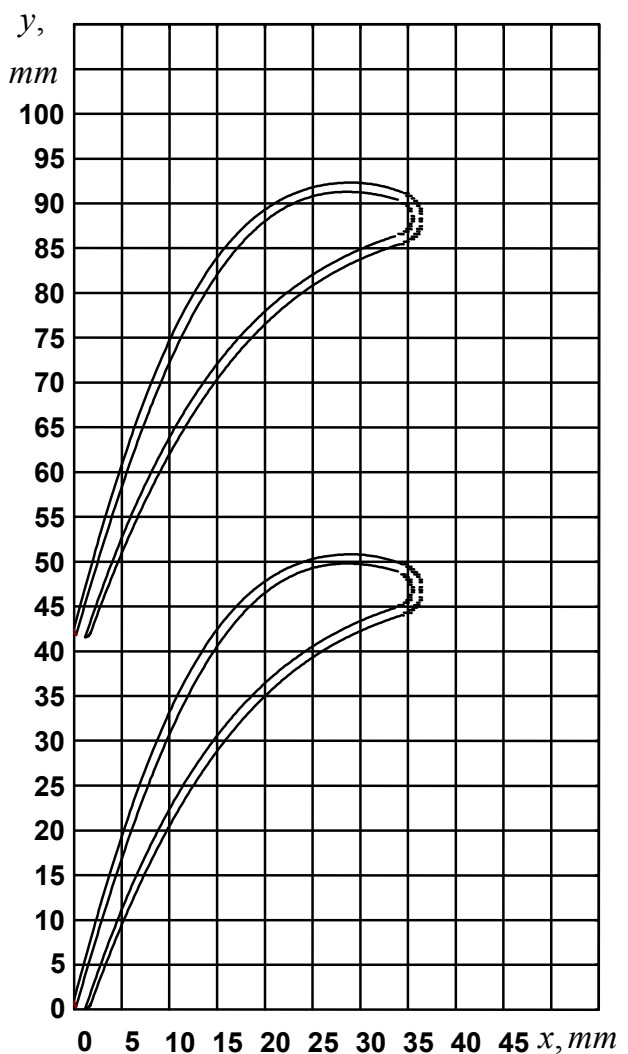

Fig 3. The cascade of profiles of the nozzle cooled blade

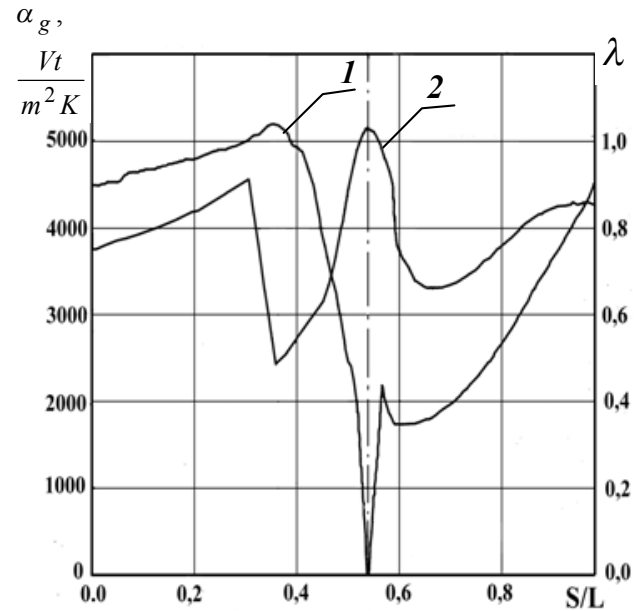

Fig 4. Distribution of the relative speeds $\lambda \quad$ (1) and of gas convective heat exchange coefficients $\alpha_{\Gamma}$ (2) along the periphery of the profile contour

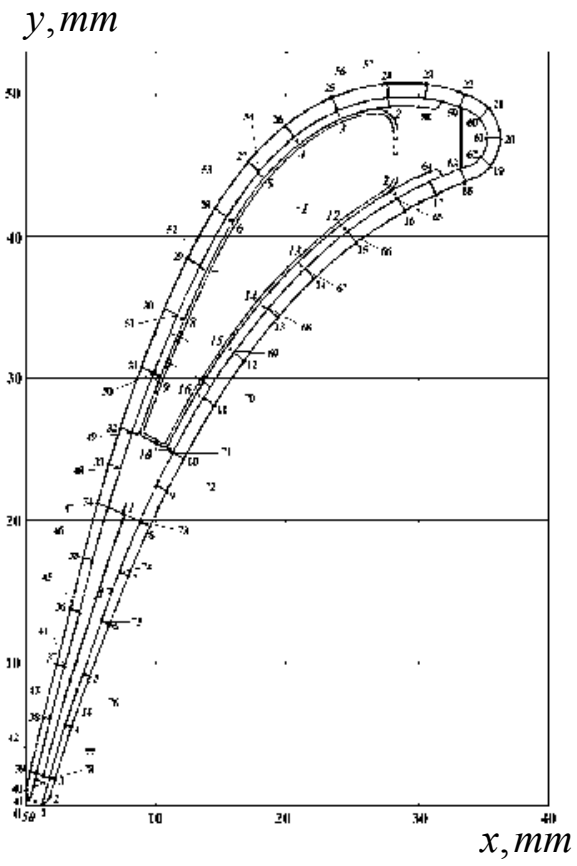

Fig 5. Geometrical model with foliation of design points of contour (1-78) and equivalent hydraulic schemes reference sections (1-50) of the experimental nozzle blade

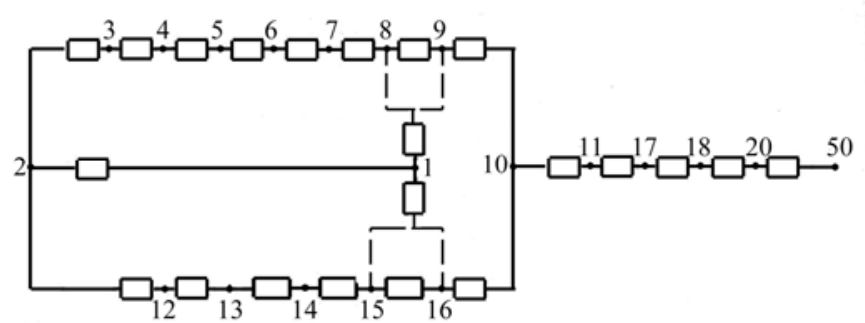

Fig 6. The equivalent hydraulic scheme of experimental nozzle blade cooling system

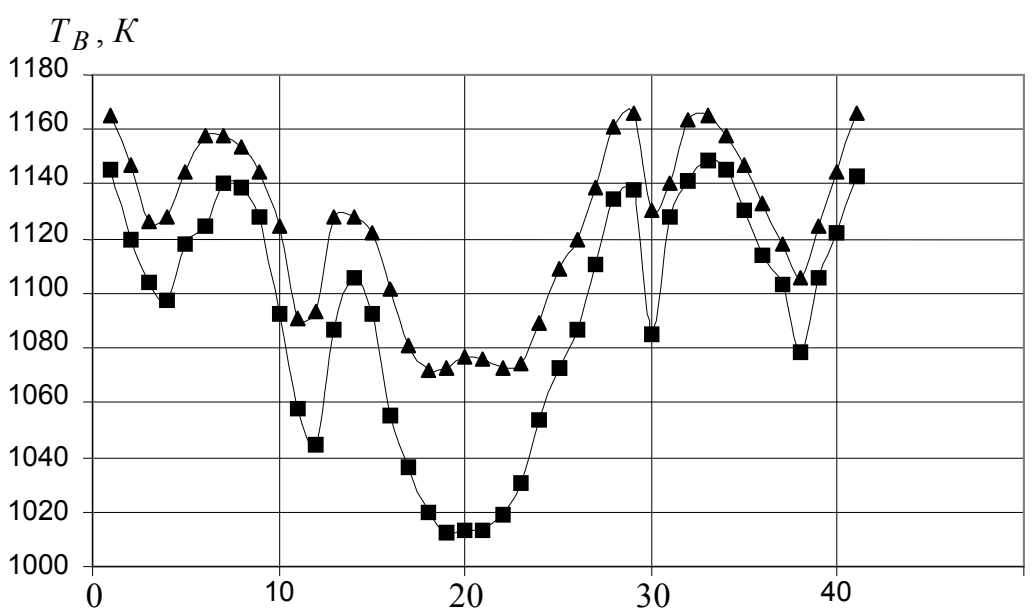

Fig 7. Distribution of temperature along outside $(\boldsymbol{\Lambda})$ and internal $(\boldsymbol{\square})$ contours of the cooled nozzle blade 


\section{Conclusions}

The reliability of the methods was proved by experimental investigations heat and hydraulic characteristics of blades in "Turbine Construction" (Laboratory in St. Petersburg, Russia). Geometric model, equivalent hydraulic schemes of cooling tracks have been obtained, cooler parameters and temperature field of "Turbo machinery Plant" enterprise (Yekaterinburg, Russia) gas turbine nozzle blade of the 1st stage have been determined. Methods have demonstrated high efficiency at repeated and polivariant calculations, on the basis of which has been offered the way of blade cooling system modernization.

The application of perfect methods of calculation of temperature fields of elements of gas turbines is one of the actual problems of gas turbine engines design. The efficiency of these methods in the total influences to operational manufacturability, reliability of engine elements design, and on acceleration characteristics of the engine.

\section{References}

1. Abasov M.T., Sadiqov A.H., Aliyarov R.Y. Fuzzy neural networks in the system of oil and gas geology and geophysics // Third International Conference on Application of Fuzzy Systems and Soft computing/ Wiesbaden, Germany, 1998. - 1998. - P.108-117.

2. Arseniev L.V., Mitryayev I.B., Sokolov N.P. The flat channels hydraulic resistances with a system of jets in a main stream // Energetika. 1985. -No 5. -P.85-89.

3. Beknev V.S., Epifanov V.M., Leontyev A.I. et al. Fluid dynamics. A mechanics of a fluid and gas. - Moscow: MGTU N.E. Bauman, 1997. $671 \mathrm{p}$.

4. Fuzzy sets, neural networks and soft computing. VAN Nostrand Reinhold /Ed. R.R Yager., L.A. Zadeh. - N. -Y., 1994. - No 4.
5. Galicheiskiy G. A thermal guard of blades. M.: MAI, 1996.

6. Hassoun M.H. Fundamentals of - The MIT press Cambridge, Massachusetts, London, England, 1995.

7. Kopelev S.Z., Slitenko A.F. Construction and calculation of GTE cooling systems. - Kharkov: Osnova, 1994. - 240 p.

8. Pashaev A.M., Sadiqov R.A., Hajiev C.M. The BEM Application in development of Effective Cooling Schemes of Gas Turbine Blades $/ / 6^{\text {th }}$ Bienial Conference on Engineering Systems Design and Analysis, Istanbul, Turkey, July, 811, 2002. - 2002.

9. Pashaev A.M., Sadiqov R.A., Iskenderov M.G. et al. The effeciency of potential theory method for solving of the tasks of aircraft and rocket design // 10-th National Mechanic Conference. Istanbul, Turkey, July 1997. - Istanbul, 1997. P. 61-62.

10. Pashaev A.M., Sadykhov R.A., Samedov A.S. et al. The solution of fluid dynamics direct problem of turbomachines cascades with integral equations method // Proceed. NAA. - Baku, 2003. - Vol 3.

11. Pashaev A.M., Sadykhov R.A., Samedov A.S. Highly effective methods of calculation temperature fields of blades of gas turbines $\mathrm{V}$ International Symposium an Aeronautical Sciences "New Aviation Technologies of the XXI century". A collection of technical papers. Section N3. Zhukovsky, Russia, August 1999. Zhukovsky, 1999.

12. Sadykhov R.A., Samedov A.S. Modelling of temperature fields of gas turbines elements. Scientists of a slip AzTU. - Baku, 1998. - Vol VI, No 5. - P. 234-239.

13. Zisina-Molojen L.N et al. Heat exchange in turbomachines. - M., 1974. 